\title{
INTERACTION OF MAGNETIC FIELDS AND \\ THE ORIGIN OF PROTON FLARES
}

\author{
L. Ǩ̆IVSKÝ \\ (Astronomical Institute, Ondřejov, Czechoslovakia)
}

\begin{abstract}
A BSTRACT
The magnetic model of a proton flare is discussed, in agreement with the known characteristic features of proton-flare development, on the basis of the interaction of an emerging magnetic channel from the photosphere, and its being wedged into the field of an already existing interaction of two systems of magnetic fields of a complex active region with spots. This explains the origin of the two emission filaments following the splitting and of the 'annular' emission filament, entering the corona, connected by a fine loop structure with two split filaments in the chromosphere. The acceleration of particles and their ejection will take place in a number of created 'zero line' spaces and in spaces where magnetic lines of force are reconnected.

An attempt is made to explain some $\mathrm{H} \alpha$ phenomena in connection with proton flares, by means of interactions of magnetic fields in the chromosphere and in the lower corona.
\end{abstract}

\section{Characteristic Features of Proton Flares}

We know the following typical characteristic features of proton-flares development:

(1) Proton flares occur in groups of certain types of spots along a 'magnetic axis' on the boundary of the magnetic field of spots polarities (Bumba, 1958; Ellison et al., 1961 ) in complex multi-pole magnetic systems, especially in the case of an interaction of two or more groups of spots and their magnetic fields (Antalová, 1967).

(2) A transient occurrence of a characteristic Y-type phase in the period of an expanding flare phase (Křivský, 1963a,b) and the appearance of a 'channel' from two or more roughly parallel emission filaments (Ellison et al., 1961; Křivský, 1963b).

(3) One of the flare filaments is usually a stationary one, the second filament shows, with flares on the disc, a tangential component of movement, and, in reality, ascends at a slanting angle (Křivský, 1963b, 1964).

(4) The moving emission filament can conceal for a transient period by its movement even large spots in the group, attains in the initial stage, that is during the disruption of the Y-phase, a velocity of $10-20 \mathrm{~km} / \mathrm{sec}$, its velocity then falls down to a few $\mathrm{km} /$ sec, and finally stops altogether (Křivský, 1963b).

(5) The emerging emission filament in $\mathrm{H} \alpha$ has an annular structure, is noticeable even in projections onto the disk, and is the first type of a fine loop structure. When emerging above the limb the filament acts as an intensive ring-shaped coronal condensation,

Kiepenheuer (ed.), Structure and Development of Solar Active Regions, 465-470. (C) I.A.U. 
evident in $\mathrm{H} \alpha$ and in the Fe XIV $5303 \AA$ and $\mathrm{CaXV} 5694 \AA$ lines (Slonim, 1963; Waldmeier, 1960; Křivský, 1968).

(6) From the opening mouth of the flare 'channel' it is possible to notice the propagation of a 'disturbance' in the form of absorption or alternately also emission traces over large distances, or an absorption filament emerges from the canal. Sometimes it is 'loose', sometimes it is anchored in the shape of an arc (Křivský, 1963b; Křivský and Nešpor, 1967).

(7) The developing flare channel shows, especially in the later stages of development, a filamentary structure, joining both the flare-emission filaments moving apart from each other (second type of loop structure) (Křivský, 1968; Bruzek, 1964).

(8) In the declining stage of the flare (following the maximum brightness) large arcs and loops, noticeable on the disk in the $\mathrm{H} \alpha$ absorption, and above the limb as large loop-type prominences either appear or renew their activity (Kleczek and Krrivský, 1960). This third type of the loop structure is spatially the largest and it appears, that the anchoring of the system of loops need not lead to large spots in groups on either side.

(9) Further characters typical for flares with a powerful ejection of fast particles were compiled by Švestka (1966). These are especially: flares with ejection of cosmic or even subcosmic rays were observed in an integral light (McCracken, 1959), some parts of the flare-emission filaments touch with the umbra of large spots (Dodson and

FIG. 1. (a) A new system of magnetic channel-tube with a considerable density of plasma energy $E_{3}$ and magnetic energy $B_{3}$, identical with the flare-emission filament in $H \alpha$, emerges and is pushed between two large magnetic systems with an interaction region (with zero point $N_{0}$ ). It is a system with lines of force running in opposite direction as regards the higher placed, already created system. In the contact space of the opposite-directed magnetic fields there occurs the condensation and acceleration of plasma, and as a result, also an emission. Such spaces are dotted on the cross-section. The interconnection of magnetic lines will create roughly two systems of interaction.

(b) A more detailed picture of a further stage of development in the space of a complex interaction. On the sides of the emerging canal two new spaces of interaction, $N_{1}$ and $N_{2}$ are created (in the crosssection plane they are seen as points, in fact they are lines). This is a stage of splitting the filament of the flare in $\mathrm{H \alpha}$ into two filaments and at the same time the rise of an emerging annular system (rotor). The condensation and the acceleration of the plasma already takes place in the spaces of newly reconnected lines of force (Syrovatskij. 1966). These spaces can lie very close to the spots, which are identical with the contact of the emission filaments of the flare in Ha and with some spots' umbrae. The arrows mark the directions of the principal plasma pressures and drifts.

(c) If one of the original macrosystems, e.g. the one seen in the picture on the right-hand side, will comply with the inequality of $E_{2}+B_{2}<E_{3}+B_{3}$, then the zero point $N_{2}$ and the corresponding regions of reconnecting lines will be moved at a slanting angle into space above and behind the spot $S_{2}$. This shifting is identical with the moving flare emission filament after this is separated. Moreover, a new emerging system of a sort of magnetic rotor is created in space with condensed plasma, which in some places can produce instabilities of various kinds connected with an exceptional acceleration of particles and their penetration into coronal and interplanetary space. The large arrows drawn in interrupted lines demonstrate the predominant resulting movement. 

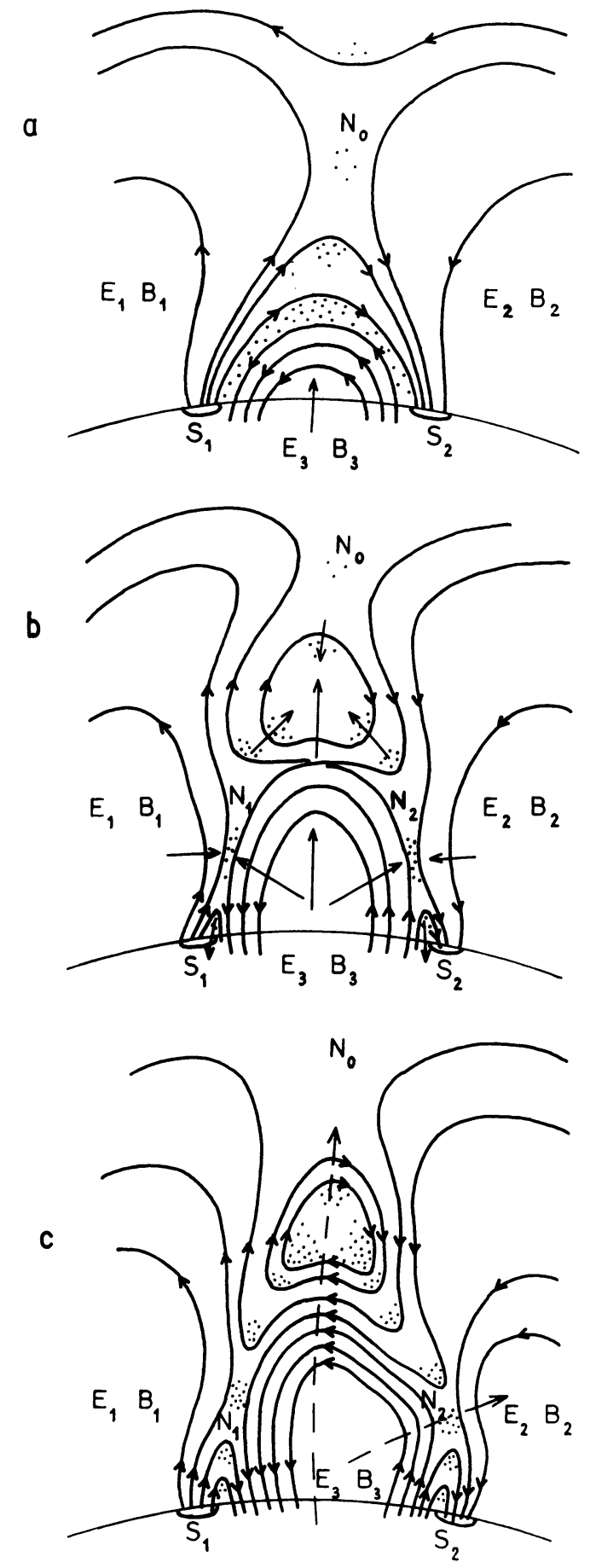
Hedeman, 1960), the impulsive very hard X-emission was radiated in the period of the Y-phase (Křivský in the paper by Švestka, 1966; Valníček, 1967).

\section{Magnetic Model of a Proton Flare}

Sweet (1956) was the first to consider the principle of the interaction of magnetic fields for the origin of flares in the space above the active areas of groups. In further works it was proved that some flares, later shown to be the flares with ejection of particles (Křivský, 1965, 1966), will occur in conjunction with the emergence of magnetic tubes from the lower regions into the chromosphere and the corona (Slonim, 1965; Kawabata, 1966; Sturrock, 1966; Banin, 1967). It was stated earlier (Krrivský, 1963b) that the walls of the emerging long and narrow tube (channel) will be identical with the flare's emission filaments. Syrovatskij (1966) asserts that radiation, i.e. a visible flare, might occur in spaces of strong condensation of plasma in a system of an interaction of two magnetic fields and in spaces of reconnected lines of force.

Due to the mentioned observed facts, listed in points (1)-(9), we submit for discussion the model of the magnetic field's interaction during the origin and development of proton flare with initial phase Y (Křivský, 1963a) and with the following phase in the shape of a channel, evident roughly from parallel emission filaments of the flare (Ellison et al., 1961; Reid, 1965).

The new system of a narrow magnetic tube emerges from the lower levels into a region of an already existing interaction of two systems of magnetic fields (vertical cross-section in Figure 1a), the considered directions of magnetic lines of forces are important for the origin of the 'zero points' (in fact, in space these are 'zero lines') and are marked with arrows. The areas of condensation and acceleration of plasma are identical with the radiation (luminous region), and are dotted. This process is identical with the origin of the long narrow-emission filament of the flare. Essentially, we are considering the emerging of the tube's magnetic system into the centre of a system of a shape used by Wild (1963).

The emerging magnetic tube (channel) is further wedged and pushed into the system of a preceding longer-term interaction, a complicated system of interaction and the appearance of 'zero points' takes place as shown in Figure $1 \mathrm{~b}$.

The tube walls would expand in a direction where the density of the kinetic energy of plasma $E$ and magnetic field $B$ is lower than the density of the plasma energy and of the field in the space of the new emerging tube. In this way, the emission filaments would move either in one or other direction, where the formula $E_{1,2}+B_{1,2}<E_{3}+B_{3}$ would be valid. The process would be identical with the $Y$-shape phase and with the appearance of two emission filaments. The interconnection of magnetic fields and their annihilation would lead at the top of the ascending flare tube to the origin of a new magnetic system, reminiscent of a rotatory cylinder (Figure $1 \mathrm{~b}, \mathrm{c}$ ). This process 
would be identical with the appearance and emergence of the peak annular emission filament, as seen by the author himself many times with these flares.

The transport of material in $\mathrm{H} \alpha$ would be very complicated and would be subject to the directions of the interacting magnetic fields, and its vertical cross-section would resemble the shape 8 . The possibility of this shape was considered, but for other reasons, by Banin (1966) and, in a more detailed model without taking into account the interaction of fields, by Kawabata (1966). The appearance of the top annular rotor (which is identical with the emerging emission filament and with condensation up to high altitudes) was explained by Kawabata as a result of a close, indefined, turbulent process.

During the later disintegration of this system, as a result of the conversion of a magnetic energy into an energy of an ejection of particles, and the restoration of the original shape of the fields in the higher chromosphere and the lower corona, a system of large spatial loops would be created (on the disk of the absorption arcs and loops emerging from the 'flare channel'). The process of contraction and control of dispersed particles by renewed magnetic lines of a large spatial character would already be asserted (Křivský and Nešpor, 1967). This process, occurring after the expanding phase of the flare and covering the time period of the interflare stages agree with a model sketched by Carmichael (1964, Figs. 54-4 and 54-5).

The acceleration of particles to high velocity (cosmic and sub-cosmic rays) would take place primarily in the 'zero lines' $N$ spaces (Dungey, 1956) and further, in the spaces of reconnecting lines of force (Sturrock, 1966; Syrovatskij, 1966). Their escape into space would occur at the beginning of the stage when the new tube would be wedged and pushed into the preceding system and to the creation of an ascending annular rising system (rotor). This phase of development is identical with the phase of a Y-shaped flare and the splitting of the channel into the shape of an orifice in the period of the first maximum burst on the radio waves (Křivský, 1963b), and can sometimes be accompanied by effects in $\mathrm{H} \alpha$ and in the radio spectra, which indicate the evolution and the very fast propagation of shock waves (Wild, 1963). The mechanism of Syrovatskij (1966) could be considered as an acceleration and escape mechanism for the particles.

The model presented here is based on the assumption that two main neutral regions, $N_{1}$ and $N_{2}$, have been formed. In the case when a newly created region of interaction $N_{3}$ occurs near the top of an emerging magnetic tube, two (instead of one) closed magnetic cylinders are formed. These cylinders have to be considered regions where extraordinary dense clouds of particles (eventually relativistic) together with HMwaves can be created, the waves collide under various angles. Relativistic particles should reach their largest energies at the beginning of the quickly evolving 'tearing' process mentioned above, when the gradients of magnetic fields on the regions of interaction are enormous and when the total magnetic energy involved in the process of conversion to the kinetic energy of particles is still very large. 


\section{References}

Antalová, H. (1967) Bull. astr. Inst. Csl., 17, 61.

Banin, V.G. (1966) Izv. Krym. astrofiz. Obs., 35, 190.

Banin, V.G. (1967) Izv. Sib. Izmiran, 2.

Bruzek, A. (1964) Astrophys. J., 140, 745.

Bumba, V. (1958) Izv. Krym. astrofiz. Obs., 19, 105.

Carmichael, H. (1964) AAS-NASA Symp. Phys. of Solar Flares, Washington, 451.

Dodson, H.W., Hedeman, E.R. (1960) Astr. J., 65, 51.

Dungey, J.W. (1956) Sixth I.A.U. Symp., Stockholm, Cambridge, 1958, p. 135.

Ellison, M.A., McKenna, S., Reid, J.H. (1961) Dunsink Obs. Publ., 1, 53.

Kawabata, K. (1966) Rep. Ionosph. Space Res. Japan., 20, 107.

Kleczek, J., Křivský, L. (1960) Bull. astr. Inst. Csl., 11, 165.

Křivský, L. (1963a) Nuovo Cim., X-27, 1017.

Křivský, L. (1963b) Bull. astr. Inst. Csl., 14, 77.

Křivský, L. (1964) Bull. astr. Inst. Csl., 15, 75.

Křivský, L. (1965) Bull. astr. Inst. Csl., 16, 27.

Křivský, L. (1966) Bull. astr. Inst. Csl., 17, 141.

Křivský, L. (1968) Bull. astr. Inst. Csl., 20 (in print).

Křivský, L., Nespor, Ju.I. (1967) Izv. Krym. astrofiz. Obs., 36, 98.

McCracken, K.G. (1959) Nuovo Cim., X-13, 1081.

Reid, J.H. (1965) NATO Adv. Study Inst. (Planetary and Stellar Magn).

Slonim, Ju. M. (1963) Soln. Dann. No. 4, 67.

Slonim, Ju. M. (1965) Soln. Dann. No. 1, 51.

Sturrock, P. A. (1966) Nature, 211, 695.

Svestka, Z. (1966) Space Sci. Rev., 5, 388.

Sweet, P. A. (1956) Sixth Int. Astr. Union Symp., Stockholm, Cambridge 1958, p. 123.

Syrovatskij, S.I. (1966) Astr. Zu., 43, 340.

Valniček, B. (1967) Bull. astr. Inst. Csl., 18, 249.

Waldmeier, M. (1960) Z. Astrophys., 51, 1.

Wild, J.P. (1963) The Solar Corona (Proc. Intern. Astr. Union Symp. No. 16) New York, p. 115. 